\title{
Remoción de sulfuros presentes en el agua residual del proceso de curtido mediante cavitación hidrodinámica
}

\author{
Degradation of sulfide from leather tanning \\ wastewater by hydrodynamic cavitation
}

\section{Remoção de sulfetos presentes nas águas residuárias do processo de curtimento por cavitação hidrodinámica}

\author{
Rafael Nikolay Agudelo-Valencia; Diana Paola Ovalle-González*; Luis Felipe Rodríguez-Rodriguez; \\ Gabriel de Jesús Camargo-Vargas; Leidy Yaneth Almonacid-Jimenez \\ Universidad Libre, Calle 8 No. 5-80 Bogotá, Colombia. \\ *dianap-ovalleg@unilibre.edu.co
}

Fecha recepción: 13 de diciembre de 2018 Fecha aceptación: 3 de abril de 2019

\begin{abstract}
Resumen
El sulfuro presente en el agua residual de la industria del curtido de cueros proviene de la operación de pelambre (depilado de la piel) en concentraciones que afectan significativamente la calidad del agua del cuerpo receptor debido a la toxicidad del vertimiento produciendo la mortandad de flora y fauna, responsable de la presencia de olores desagradables en el cauce y la notable disminución del oxígeno disuelto en el agua del afluente. En la presente investigación, la remoción de sulfuro es evaluada empleando la técnica cavitación hidrodinámica $(\mathrm{CH})$, un proceso de oxidación avanzada analizado desde diferentes parámetros de operación como $\mathrm{pH}$ inicial del agua residual y presión de entrada para un tiempo de reacción fijo de 90 minutos; parámetros de diseño fueron establecidos mediante el uso de dos prototipos, variando el número y diámetro de orificios en el punto de estrangulamiento con el objetivo de determinar las condiciones óptimas del reactor para el tratamiento de este tipo de contaminante en las aguas residuales. Se obtuvo una remoción máxima del 32,6 \% de la concentración inicial de sulfuro sin aplicar algún tipo de catalizador o reactivo químico; de igual modo se realizó un análisis costobeneficio debido a la eficiencia de la tecnología aplicada en comparación con la energía requerida por el reactor empleado, donde se observa una disminución del $198 \%$ del costo total del tratamiento actual. Finalmente, la cavitación hidrodinámica es una tecnología sustentable para la industria del curtido de cueros.
\end{abstract}

Palabras clave: Aguas Residuales de Pelambre; Cavitación Hidrodinámica; Curtiembre; Oxidación Avanzada; Sulfuro.

\begin{abstract}
Sulfide present in the wastewater from industrial tanneries is resulting from unhairing (depilated of the skin) in concentrations that affects significantly the water system due to its toxicity, producing the mortality of flora and fauna, responsible for the presence of unpleasant odors in the riverbed and decreasing the dissolve oxygen in the water of the receiver stream. In the present research, removal of sulfide is evaluated using hydrodynamic cavitation ( $\mathrm{HC}$ ). An advanced technology is analyzed by different parameters as initial $\mathrm{pH}$ solution and inlet pressure, set in two prototypes established for a reaction time of 90 minutes. Design parameters as number and diameter of holes in the constriction are estimated according on the degradation extent with the objective of knowing best reactor characteristics for wastewater treatment applying this technique. A maximum extent of sulfide oxidation of $32.6 \%$ was reached without using any further reagents; cost - benefit analysis had been discussed in the present investigation due to the


enhance of cavitation hydrodynamic reached compared with the energy required by the reactor observing a shortfall of $198 \%$ related with the conventional treatment, $\mathrm{HC}$ was found as a sustainable technology for tannery industries.

Keywords: Advanced Oxidation; Hydrodynamic Cavitation; Liming Wastewater; Sulfur; Tannery.

\section{Resumo}

Na presente investigação, a remoção de enxofre é avaliada usando a técnica de cavitação hidrodinâmica (HC), um processo de oxidação avançada, analisado a partir de diferentes parâmetros de funcionamento como o pH inicial da pressão de águas residuais e da entrada para um tempo de resposta fixo de 90 minutos; parâmetros de projeto foram estabelecidos através da utilização de dois protótipos, variando o número e o diâmetro dos furos no ponto de estrangulamento com o objetivo de determinar as condições ideais para o tratamento deste tipo de contaminante do reator a águas residuais. Obteve um máximo de afastamento de $32,6 \%$ da concentração inicial de enxofre sem aplicar qualquer catalisador ou reagente químico; da mesma forma um análise custo-benefício devido a eficiência da tecnologia aplicada em comparação com a energia necessária pelo reator utilizado, mostrando uma diminuição de 198\% do custo total do tratamento atual. Finalmente, cavitação hidrodinâmica é uma tecnologia sustentável para o indústria de curtimento de couro.

Palavras-chave: Águas Residuais de Calagem; Hidrodinâmica da Cavitação; Curtume; Avançado de Oxidação; Sulfídrico.

\section{Introducción}

En la actualidad, el sector dedicado a la producción de cuero, es decir, al procesamiento de piel de animal (normalmente bovino) en un material perenne que ha sido criticado debido a la magnitud del impacto ambiental que genera en el ecosistema [1-3]. El recurso hídrico es el principal afectado por este tipo de industria, puesto que es utilizado en cada una de las etapas del proceso productivo y alterado con sustancias agresivas entre las cuales se puede mencionar ácido fórmico, cloruro de sodio, sulfuro de sodio, cloruro de amonio, sulfato de amonio, sulfato de cromo entre otros $[4,5]$ compuestos que posteriormente son liberados en las aguas residuales de los distintos procesos, causando afectaciones en la flora, fauna y en la salud del ser humano como irritación cutánea y ocular [6] en caso de no recibir un tratamiento previo efectivo que garantice la calidad del vertimiento. En Colombia, la actividad industrial de curtido de pieles llegó a los municipios de Villapinzón y Chocontá a mediados de los años 50 [7] ubicándose en la sabana norte de la capital del país con 110 curtiembres que ocupan el primer lugar en producción mensual de cuero en Colombia [8,9]. Las curtiembres asentadas en el corredor vial que une a los municipios de Chocontá y Villapinzón se encuentran sobre la cuenca del río Bogotá, catalogado como uno de los más contaminados a nivel mundial [10], y para el caso de las aguas residuales del sector curtidor, es preciso señalar que le aportan un alto potencial contaminante, caracterizado por altas concentraciones de sólidos, materia orgánica carbonácea y nitrogenada, sulfuros y compuestos de amonio.

Una de las actividades principales que se debe realizar en el curtido de pieles, es la de remover el pelo de la piel animal para poder continuar el proceso. A esta actividad se le llama pelambre o depilado, y para lograr el decapado de la piel, se utiliza sulfuro de sodio, dando lugar a la presencia de sulfuro en las aguas residuales del proceso. El sulfuro, mejor llamado hidrogeno de sulfuro, es un ácido diprótico y en las aguas residuales del proceso de pelambre se encuentra presente tanto en forma de $\mathrm{H}_{2} \mathrm{~S}$, $\mathrm{S}^{2-}$ y $\mathrm{HS}^{-} \mathrm{y}$, aunque el sulfuro o azufre se encuentra presente en la mayoría de los ecosistemas acuáticos, en el caso de las aguas residuales de pelambre o decapado puede ser vertido a concentraciones del orden de $1500 \mathrm{mg} \mathrm{L}^{-1}$ $[11,12]$. Este contaminante es tóxico y precursor de serios problemas que acarrean mortandad de flora y fauna, así como también da lugar a olores desagradables en el cauce que en casos extremos causan irritación cutánea y ocular por citar algunos $[6,13]$ social y económico de la industria de curtido y preparado de cueros en Colombia, el Ministerio de Ambiente, Vivienda y Desarrollo TerritorialMAVDT, en asocio con el Instituto de Hidrología, Meteorología y Estudios Ambientales- IDEAM y 
con la colaboración de la Mesa Nacional del Sector de Curtido y Preparado de Cueros, elaboraron este documento como instrumento orientador a las pequeñas, medianas y grandes empresas del sector, en el uso de medidas de prevención, mitigación, tratamiento y valoración de sus impactos ambientales y sociales. La guía está compuesta por seis (6). Por otro lado, dependiendo del estado de oxidación del azufre, de la concentración y del tipo de exposición (ingesta, inhalación, contacto con ojos y piel), los compuestos de equilibrio del sulfuro pueden generar irritación en fosas nasales, edema pulmonar, convulsiones, inconsciencia e incluso pueden ocasionar la muerte [14]. Además, genera corrosión en las tuberías de desagüe [15] y condiciones anaerobias puesto que disminuye el oxígeno disuelto en el cauce, generando a su vez, que este pierda la calidad del servicio ambiental para lo cual está destinado.

Parámetros como la demanda química de oxígeno ( $\left.\mathrm{mg} \mathrm{L}^{-1}\right)$, cloruros ( $\mathrm{mg} \mathrm{L}^{-1}$ de $\mathrm{Cl}$ ), $\mathrm{pH}$, oxígeno disuelto $\left(\mathrm{mg} \mathrm{L}^{-1}\right)$ y sulfuros $\left(\mathrm{mg} \mathrm{L}^{-1}\right)$ son relacionados directamente con la actividad del procesamiento de cuero [16]. La normatividad colombiana ha establecido altos estándares para los parámetros de control del vertimiento de aguas residuales provenientes de curtiembres sobre la cuenca del río Bogotá debido a la gran población de industrias presentes a lo largo del cauce, fijando para sulfuros verter máximo $1 \mathrm{mg}$ por cada litro de agua residual [17], reto bastante ambicioso, tanto para la autoridad ambiental como para la comunidad científica.

El tratamiento convencional de las aguas residuales en una curtiembre para el agua procedente de la etapa de pelambre, consiste en airear el agua alrededor de 14 horas para lo cual se hace uso de un soplador o blower, que generalmente cuenta 5,220 $\mathrm{W}$ de potencia, forjando un ambiente oxidante en el agua residual; sin embargo, no es suficiente para remover la considerable cantidad de sulfuro presente, por lo cual a esta operación es necesario agregarle manganeso en forma de sulfato de manganeso, catalizador que acelera el proceso, dejando una concentración final de sulfuros alrededor de los $150 \mathrm{mg} / \mathrm{L}$ en 10 horas de oxidación. Por otro lado, es preciso mencionar que los costos del tratamiento de solamente este tipo de agua son bastante elevados, según los datos recolectados en las empresas que permitieron el desarrollo del presente trabajo, el valor del tratamiento alcanza los $\$ 2,32$ USD por cada $\mathrm{m}^{3}$ de agua residual tratada, sin contar las aguas residuales procedentes de las otras actividades del proceso productivo. Para concluir, el tratamiento actual que se realiza en las curtiembres para el agua de pelambre deja varias desventajas para el gremio, puesto que, si bien no logra el objetivo de remover lo requerido por la normativa, genera sulfatos no solo por la reacción de oxidación sino por la adición del catalizador, el costo del tratamiento es alto, lo que da lugar a la búsqueda de nuevas alternativas que satisfagan tanto la normatividad vigente, la protección del ecosistema y represente una alternativa de tratamiento amigable para la economía del sector industrial.

El propósito de la presente investigación es evaluar la cavitación hidrodinámica $(\mathrm{CH})$ como alternativa de tratamiento para el agua residual de pelambre, con el fin de determinar la disminución de la concentración de sulfuro en un tiempo determinado de experimentación, identificando si dicho proceso es sostenible para el sector curtidor. La CH es una de las técnicas de oxidación avanzada, AOP por sus siglas en inglés (Advanced Oxidation Process), que consisten en la producción de radicales hidroxilos para la oxidación de los contaminantes y opera bajo condiciones (temperatura, presión, $\mathrm{pH}$ ) específicas según la sustancia a tratar [1820]. Este tipo de tecnología se ha desarrollado a partir de la década de los 70 y ha alcanzado una amplia aceptación a nivel mundial por su eficiencia [21]. La CH es una tecnología verde relativamente nueva que promueve, a través de una caída súbita de presión y asimismo, el aumento de la temperatura, la degradación, remoción y/o mineralización de sustancias presentes en el agua [22] y consiste en una oxidación acompañada por la producción de radicales $\mathrm{OH}^{*}$, ocasionados por la variación de la velocidad y presión del fluido, lo cual favorece la formación, crecimiento e implosión de microburbujas en el seno del líquido $[21,23]$ de manera que se presenta una mezcla gas-líquido, de modo que dentro de las burbujas se presentan puntos críticos de temperaturas muy altas, dependiendo de la naturaleza de los gases contenidos en el líquido, dando lugar a gradientes de temperatura de hasta 10,000 K [23-25]. En la aplicación de la cavitación hidrodinámica en un reactor experimental, se debe tener en cuenta que la generación de microburbujas o cavidades, precursoras del efecto y el cambio súbito de presión, son generadas por una reducción de área en la geometría de la tubería. En algunos casos, como Padoley et al. [24], Bagal y Gogate [18] y Saharan et al. [26], se ha empleado un sistema 
venturi para generar la caída de presión, mientras que en la mayoría de casos [27-30], se emplea una placa perforada con cierto número de orificios, tal y como se realizó en la presente investigación, debido a que el dispositivo utilizado simplemente requiere el cambio de las placas perforadas para modificar el área libre permitido para el flujo del líquido, puesto que a menor área la intensidad de la cavitación incrementa y asimismo aumentan sus beneficios [31,32]. De igual manera, es importante mencionar que la geometría del orificio en la placa perforada ha sido estudiada por varios autores como Balasundaram y Harrison [33], entre otros [34,35], y han concluido que el orificio debe ser circular puesto que dan lugar a condiciones más favorables de degradación de los contaminantes estudiados. El presente trabajo busca aplicar la cavitación hidrodinámica como técnica de oxidación avanzada al sulfuro, contaminante altamente perjudicial como se ha descrito previamente y establecer parámetros óptimos para su degradación.

\section{Materiales y métodos}

\section{Materiales}

El agua residual del proceso de pelambre o depilado de pieles fue recolectada de la Curtiembre Comercializadora Caribú ubicada en el Municipio de Villapinzón, Cundinamarca (Colombia). Una vez el proceso de pelambre o decapado de la piel finalizó en la curtiembre, el agua residual se recolectó y almacenó en un contenedor de polietileno con capacidad de $1000 \mathrm{~L}$, el tiempo máximo de almacenamiento del agua fue de 6 horas. Las muestras de agua residual tomadas a lo largo de los ensayos fueron almacenadas en frascos color ámbar y boca ancha, fabricados en polietileno de alta densidad y con capacidad de 0,09 L, con tapón de rosca para evitar la entrada o salida de aire. Los demás insumos o reactivos químicos empleados fueron soluciones de hidróxido de sodio $(\mathrm{NaOH}) 6 \mathrm{~N}$ y ácido clorhídrico (HCl) $6 \mathrm{~N}$. Estos dos reactivos se emplearon para realizar el ajuste de $\mathrm{pH}$ inicial del agua residual según el requerimiento del ensayo a realizar, previo al inicio del ensayo. Todos los reactivos empleados fueron grado analítico Merck Millipore. Para la realización de las diluciones se hizo uso de agua ultra pura obtenida en un purificador de agua ELGA que proporciona agua Tipo II, es decir de conductividad con concentraciones de COT entre 50 y $60 \mathrm{ppb}$, el cual suministra agua con una conductividad menor a $0,2 \mu \mathrm{S} \mathrm{cm} \mathrm{cm}^{-1}$.

\section{Diseño experimental}

El cavitador hidrodinámico fue diseñado a modo de sistema cerrado con recirculación. La bomba se conecta al fondo del tanque y se hace circular el agua residual a través del sistema de reducción de área para lograr la cavitación y retornar el agua al taque. Se construyeron dos prototipos: el primero se representa Figura $1(\mathrm{~A}-1)$. Este modelo incluye un tanque de almacenamiento de $65 \mathrm{~L}$ de capacidad, una bomba regenerativa de $372,85 \mathrm{~W}$ de potencia, $343,22 \mathrm{kPa}$ de presión y diámetro de succión y descarga de $2,54 \mathrm{~cm}$. Esta bomba puede suministrar un caudal máximo de $0,5 \mathrm{~L} \mathrm{~s}^{-1}$. El segundo prototipo fue conformado por un tanque con capacidad de 1000 L llenado al $60 \%$. La bomba para hacer circular el agua residual posee una potencia de $1471 \mathrm{~W}$, con 539,35 kPa de presión que puede suministrar un caudal máximo de $0,37 \mathrm{~L} \mathrm{~s}^{-1}$. Continuando con el diseño del sistema, la descarga del agua sucede de forma vertical y una vez el fluido es expulsado de la bomba, se encuentra con un manómetro tipo bourdon con margen de error de $\pm 0,5 \%$ de la escala, seguido por una placa perforada hecha en bronce, en la cual la reducción de área de las perforaciones ocasionan la cavitación hidrodinámica, como se evidencia en la Figura 1 (B), representando una reducción del área transversal de flujo del $78 \%$ ( $\mathrm{B}-1$ utilizada para el primer prototipo) y $90 \%$ ( $\mathrm{B}$ - 2 para el segundo prototipo) con 2 y 3 orificios respectivamente, cada uno de $4 \mathrm{~mm}$ de diámetro. El manómetro inicial, la placa y un manómetro que mide la presión después de la contracción están sujetados por una tubería plástica transparente de $0,5 \mathrm{~m}$ de longitud y una pulgada de diámetro, como se puede observar en la Figura $1(A-2)$, con el objetivo de visualizar las burbujas formadas a partir del efecto de cavitación hidrodinámico que se forma por la contracción. Finalmente, el ducto transparente es conectado a una manguera espiralada de material PVC que conduce el agua sin generar cambios bruscos de dirección en el recorrido y de ese modo favorecer que la velocidad de flujo en el ducto sea constante. 


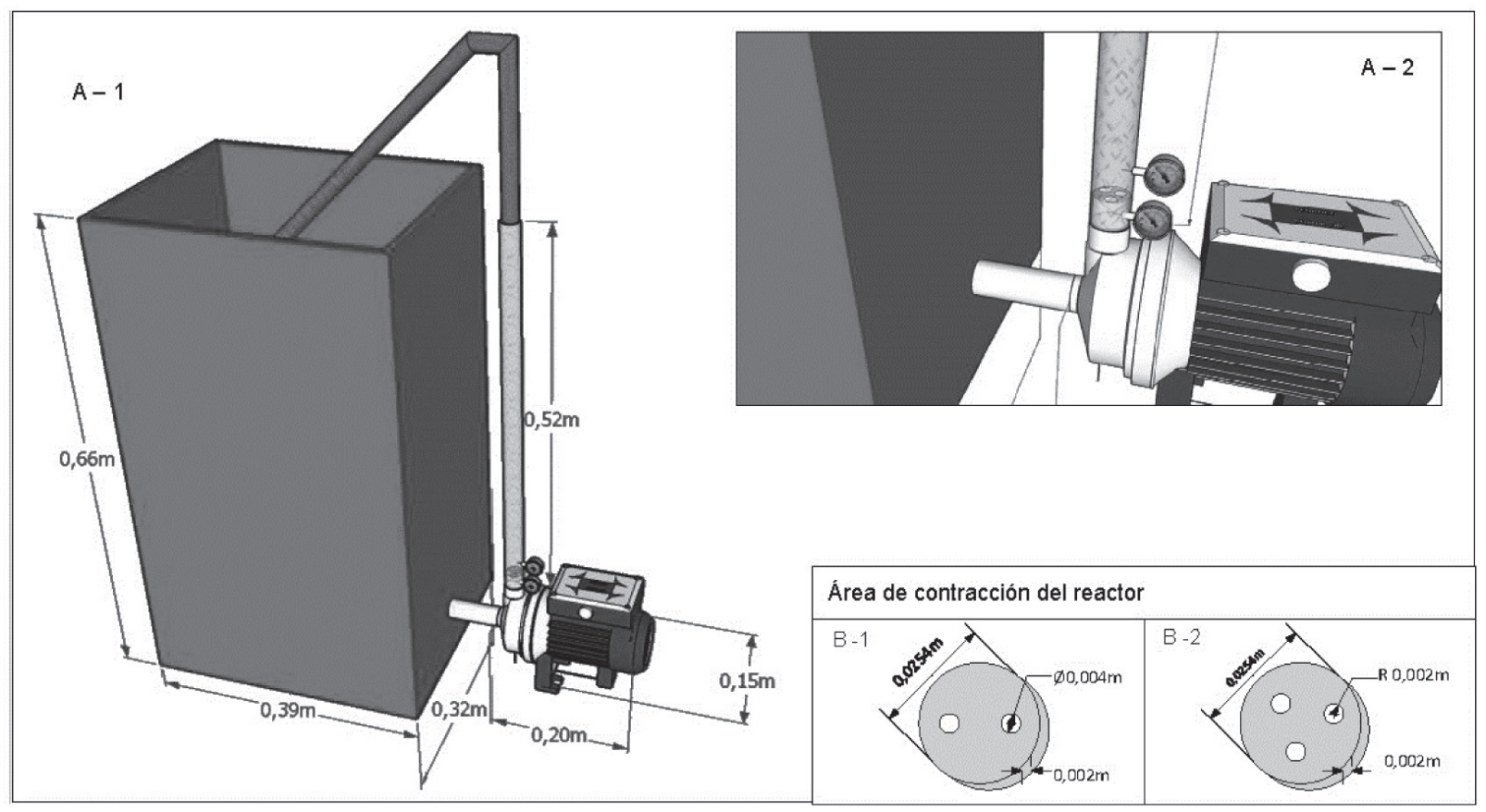

Figura 1. Esquema del diseño del cavitador hidrodinámico.

\section{Metodología experimental}

Cada experimento fue realizado en campo, es decir, en las instalaciones de la curtiembre Comercializadora Caribú. La duración de cada ensayo fue de 90 minutos con el fin de someter al proceso de cavitación hidrodinámica $50 \mathrm{~L}$ de agua residual en el primer prototipo y $600 \mathrm{~L}$ para el segundo prototipo. El agua utilizada para los ensayos no fue tratada previamente, es decir, el agua no tuvo un proceso de filtración previo para retirar solidos suspendidos. Ademas, se realizó toma de muestras y aforo de caudal cada 15 minutos, para un total de 7 muestras por ensayo. Los tanques no contaban con sistema de ajuste de temperatura y el $\mathrm{pH}$ inicial de la muestra de agua residual fue ajustado con $(\mathrm{HCl})$, o $(\mathrm{NaOH})$ según fuera el caso. Para determinar el pH se empleó un multiparámetro microprocesador portátil marca Hatch. Las variables analizadas fueron la potencia de la bomba, teniendo variación de 745,7 W (0,5 $\mathrm{hp}$ ) y $1471 \mathrm{~W}(2 \mathrm{hp}$ ) y el $\mathrm{pH}$ inicial del agua residual, el cual fue variado entre 10,0 y 14,0 .

Las muestras fueron conservadas bajo el protocolo establecido, siguiendo las instrucciones que el método empleado sugiere. Cada muestra fue debidamente rotulada y almacenada en un lugar oscuro a $7{ }^{\circ} \mathrm{C}$ de temperatura y transportadas al laboratorio. El tiempo máximo de almacenamiento fue de tres días antes de ser analizadas. Cada ensayo tuvo dos réplicas, es decir que se realizaron tres ensayos por cada juego de variables designada.

\section{Métodos analíticos}

Las muestras fueron tomadas y almacenadas según lo diseñado, para luego ser analizadas en el laboratorio de química analítica de la Universidad Libre de Colombia. Para iniciar los análisis, las muestras fueron sometidas a centrifugación durante 10 minutos con una velocidad de 60 RPM, a fin de eliminar interferencias en el método seleccionado para el análisis de sulfuros. La concentración de sulfuros fue determinada empleando el método No. 4500-S2- F consignado en el libro Standard Methods for the Examination of Water and Wastewater [36]. Las demás variables, fueron analizadas conforme a los protocolos definidos en el libro señalado previamente.

El número de muestras a tomar por cada ensayo fue establecido tomando como base la bibliografía consultada, teniendo en cuenta que en la mayoría de ensayos en los cuales se emplea cavitación hidrodinámica se toma un tiempo entre 60 minutos y 120 minutos [26,37], por lo cual se adoptó un tiempo en el intervalo previamente hallado (90 $\mathrm{min}$ ) 
y se decidió tomar muestra cada 15 minutos debido a que facilita la lectura en la gráfica de tendencia.

\section{Resultados y discusión}

\section{Efecto del pH inicial}

El pH inicial de la solución o fluido es un parámetro de alta relevancia para la degradación de contaminantes químicos en el agua, en especial cuando el contaminante puede existir en el medio acuoso en tres formas solubles, sulfuro de hidrogeno $\left(\mathrm{H}_{2} \mathrm{~S}\right)$, ion bisulfuro ( $\left.\mathrm{HS}^{-}\right)$y el ion sulfuro $\left(\mathrm{S}^{2-}\right)$ [38]. El pH de la solución determina el equilibrio de las especies de sulfuro en el agua residual, para pH menor de 7,5 (Ecuación 1). En el fluido predominará la especie $\mathrm{H}_{2} \mathrm{~S}$, única capaz de pasar de estado acuoso a fase gaseosa quedando libre en la atmosfera [38], siendo causante de olores fuertes y desagradables, además de afectar la salud humana, hecho por el cual se debe evitar el tratamiento de este tipo de agua residual a $\mathrm{pH}$ ácido o menor a 7,5. Para pH mayor de 7,5, prevalecen en el equilibrio las especies que tienen iones negativos libres, facilitando la oxidación de estas, de modo que el azufre pueda llegar a un estado oxidativo +6 , es decir, prevalece la formación de ion sulfato en el medio (Ecuación 2). La concentración inicial de sulfuros en el agua residual empleada para los ensayos de tratamiento fue de $1650 \mathrm{mg} \mathrm{L}^{-1}$.

$$
\begin{gathered}
\mathrm{H}_{2} \mathrm{~S}+\mathrm{H}_{2} \mathrm{O}_{2} \rightarrow 2 \mathrm{H}_{2} \mathrm{O}+\mathrm{S}^{0} \text { para } \mathrm{pH}<7,5 \\
\mathrm{H}_{2} \mathrm{~S}+4 \mathrm{H}_{2} \mathrm{O}_{2} \rightarrow \mathrm{SO}_{4}{ }^{2-}+4 \mathrm{H}_{2} \mathrm{O}+2 \mathrm{H}^{+} \text {para } \mathrm{pH}>7,5
\end{gathered}
$$

Los ensayos fueron llevados a cabo a $\mathrm{pH}$ entre 10,0 y 14,0 , considerando el ultimo valor como el más próximo al $\mathrm{pH}$ característico del agua residual de pelambre/decapado. Como se puede observar en la Figura 2, después de 90 min de cavitación hidrodinámica, se evidencia que a $\mathrm{pH}$ 14,0 , el porcentaje de remoción de sulfuros es más elevado $29,4 \%$ para el tratamiento en el primer prototipo y $32,6 \%$ para el segundo prototipo, en comparación con los ensayos realizados a $\mathrm{pH}$ de 10,0 , para el cual los porcentajes de remoción de sulfuro para el primer y segundo prototipo fueron del $10,7 \%$ y $30,1 \%$ respectivamente, de manera que es valido indicar que la remoción de sulfuro presenta una clara dependencia del $\mathrm{pH}$ del agua residual y se favorece la oxidación de sulfuro a $\mathrm{pH}$ alto, hecho que se aprecia para los dos prototipos empleados en los ensayos.
Por otra parte, es importante resaltar que el efecto observado del $\mathrm{pH}$ se ha reportado en otras investigaciones $[18,20,39]$ en las cuales se reporta que la eficiencia de remoción de los contaminantes por $\mathrm{CH}$ varía en función del $\mathrm{pH}$. Algunas sustancias se degradan mejor en condiciones básicas, mientras que para otras sustancias, como por ejemplo el plaguicida Metil Paratión [20], es preferible realizar el tratamiento en un medio ácido.

\section{Efecto de la presión del sistema}

Con relación a la potencia aplicada en cada prototipo, el efecto que esta tiene sobre la concentración de sulfuro en el agua residual se presenta en la Figura 2. Se observa que, a mayor potencia de la bomba, la concentración de sulfuros disminuye en mayor proporción, es decir aumenta la remoción de sulfuros. Sin embargo, no es significativo el cambio en la concentración observado para el ensayo realizado a $\mathrm{pH} 14,0$ en el prototipo 1 (372,85 kW) con respecto a la concentración final para el ensayo a pH 10,0 en el prototipo 2 (1471 kW). La concentración final de sulfuros en estos ensayos fue de $1164,5 \mathrm{mg} \mathrm{L}^{-1}$ y $1154 \mathrm{mg} \mathrm{L}^{1}$, respectivamente, de manera que el aumento de la potencia aplicada compensa el efecto del $\mathrm{pH}$ en la oxidación de sulfuros debido a los resultados observados donde el delta de diferencia de remoción es de 10,5 mg de sulfuro, hecho que sustenta lo observador por Thanekar et al. en la degradación de cabamazepina empleando cavitación hidrodinámica [40]. De igual manera, se evidencia la relación de la presión de entrada con la remoción del contaminante, de modo que a mayor presión de entrada se favorece la cavitación hidrodinámica en el medio, debido a que las cavidades o microburbujas implotan de forma más violenta generando mayor cantidad de radicales hidroxilos que fortalecen el ambiente oxidante en el medio, llegando a una mínima concentración de sulfuros en el agua de $1112,6 \mathrm{mg} \mathrm{L}^{-1}$ para el ensayo ejecutado en el segundo prototipo $(539,35 \mathrm{kPa})$ y a $\mathrm{pH} 14,0$. Varios autores han reportado el efecto de la presión de entrada sobre la degradación de diferentes contaminantes $[20,24]$ sin obtener una presión óptima que maximice de forma significativa los beneficios de la técnica. Por lo tanto, el pH del agua residual es el parámetro que predomina la eficiencia de reacción. Los resultados indican que existe una interacción con la presión aplicada al sistema y dicha presión. 


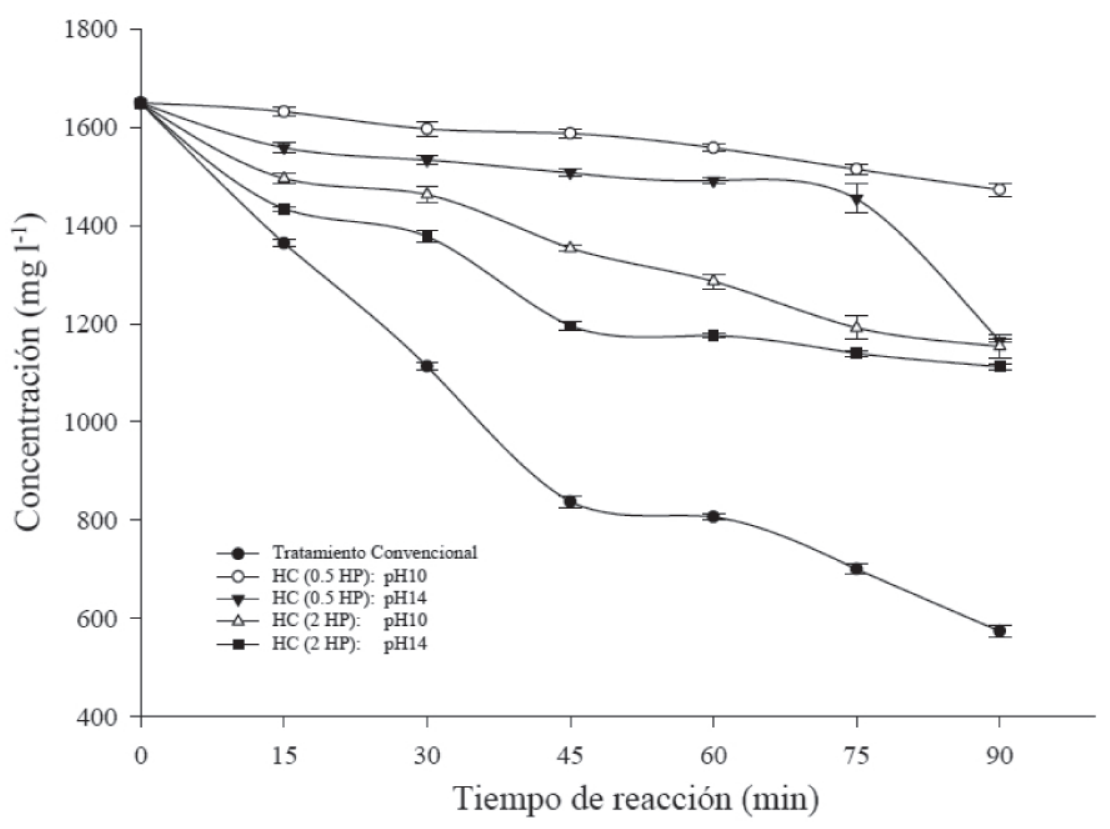

Figura. 2. Efecto del pH en relación con la potencia del sistema para la degradación de $1650 \mathrm{mg} \mathrm{L}^{-1}$ de sulfuro.

\section{Análisis de las características hidráulicas}

En los prototipos empleados, la cavitación hidrodinámica es inducida por medio de una placa perforada que reduce el área transversal, el número de orificios en cada placa brindan la posibilidad de maximizar la cavitación cuando se incrementa el número y se reduce el área de estos [29]. Las características hidráulicas del aparato son estudiadas para corroborar la eficiencia de la cavitación hidrodinámica inducida por medio de parámetros de diseño como el caudal, número y área de orificios, presión de entrada y salida, entre otros. El número de cavitación es uno de los parámetros adimensionales de mayor uso puesto que define que tan favorables son las condiciones del sistema para que ocurra la cavitación en condiciones óptimas, y se define en la ecuación 3 , para la cual es la presión después del punto de cavitación, es la presión de vapor del líquido y es la velocidad de flujo a través del orificio [23].

$$
\sigma_{a}=\frac{\left(P_{2}-P_{V}\right)}{\frac{\rho v_{o}^{2}}{2}}
$$

El efecto del número de cavitación en la técnica aplicada toma lugar cuando el líquido atraviesa el área del plato o placa perforada, de modo que una fracción del fluido tiende a pasar a fase vapor debido a la súbita caída de presión, dando lugar a la formación e cavidades o burbujas de vapor, de modo que la cavitación resulta proporcional a la velocidad con que el líquido atraviesa el punto de reducción de área de flujo, es decir, el número de orificios y el diámetro de estos son de alta importancia para maximizar el efecto de la cavitación. Por lo tanto, a pesar que el número de cavitación por sí solo no es un parámetro [41], se puede concluir que a menor número de cavitación, es mayor la intensidad de la cavitación en el sistema, generando mejores resultados en la remoción de contaminantes como se aprecia en los reportes realizados por Sivakumar y Pandit [37], y otros investigadores [18,42], los resultados obtenidos por estos investigadores indican mayor degradación para valores bajos del número de cavitación. En el presente estudio, para el prototipo 2 , el número de cavitación alcanza un valor de 0,33 , resultado favorable en comparación con el número de cavitación alcanzado en el prototipo $1(0,76)$ y puede ser atribuido a la diferencia del área transversal entre uno y otro. Por otro lado, Badve et al. [29] señalan que la cavitación es ideal a números de cavitación igual o menores a 1 , y aunque el número de cavitación es un parámetro decisivo para el diseño del sistema de tratamiento, el efecto de cavitación hidrodinámica es la razón por la cual la oxidación del contaminante toma lugar en el medio. Otro parámetro relevante es el número de ciclos que se realizó por cada ensayo, es decir, el 
número de veces que la totalidad del agua pasó a través de la placa perforada o punto de cavitación, siendo de 6 y 9 veces el número de pasos para el prototipo 1 y el prototipo 2, respectivamente. Debe indicarse que a mayor número de veces que el agua es recirculada a través de la zona de cavitación, el líquido entra en contacto con mayor cantidad de radicales hidroxilos, de modo que se incrementa el efecto de la cavitación sobre la degradación de contaminantes, por lo cual, los resultados obtenidos en la presente investigación pueden ser atribuidos de igual manera al número de ciclos de cavitación, puesto que la remoción es mayor en ambos casos de $\mathrm{pH}$ para el prototipo 2, prototipo para el cual el número de ciclos fue mayor.

Tabla 1. Características de flujo y números de cavitación.

\begin{tabular}{ccccc}
\hline Prototipo & $\mathbf{Q}_{\mathrm{m}}(\mathbf{L} / \mathbf{s})$ & $\boldsymbol{v}_{\boldsymbol{o}}(\mathbf{m} / \mathbf{s})$ & No. de ciclos & No. cavitación $\left(\boldsymbol{\sigma}_{\boldsymbol{a}}\right)$ \\
\hline 1 & 0,071 & 1,88 & 6 & 0,76 \\
2 & 1 & 39,79 & 9 & 0,33 \\
\hline
\end{tabular}

* Temperatura de referencia $15^{\circ} \mathrm{C}$.

En la Tabla 2 se pueden observar $\alpha$ y $\beta$, parámetros característicos para cuantificar el efecto de la geometría del sistema, relacionados directamente con el número de orificios y el diámetro de cada uno de ellos. Para el presente documento se trabajan orificios circulares de un mismo diámetro, únicamente cambia el número de orificios empleados en cada prototipo.

El parámetro a toma en cuenta el perímetro total de los orificios en relación con el área total abierta de la placa perforada [43], tal como se evidencia en la ecuación 4. a tiene unidades de $\mathrm{mm}^{-1}$, y el valor de este parámetro fue de $1 \mathrm{~mm}^{-1}$ para los dos prototipos. Otros investigadores han obtenido valores mínimos de mínimo 0,8 $\mathrm{mm}^{-1}$ y máximo de $4 \mathrm{~mm}^{-1}[37,43]$, con similares diámetros de operación, de lo cual se puede concluir que es debido a que el diámetro no cambia de valor $(4 \mathrm{~mm})$. En todos los casos, a permanece constante, independientemente del área total de los orificios, que asimismo es dependiente del número de orificios, debido a que Huang et al. [43] evalúa el sistema con una placa de igual diámetro pero con solo un orificio y obtiene el mismo valor $\left(1 \mathrm{~mm}^{-1}\right)$. El mismo estudio concluye que a mayor valor de $\alpha$ incrementa la tasa de remoción del contaminante empleando la cavitación hidrodinámica. Por otro lado, se ha demostrado que el diámetro afecta la frecuencia de la turbulencia en la zona de cavitación [44], siendo más intensa la cavitación para altos valores de este parámetro.

$$
\begin{gathered}
\alpha=\frac{\text { Perímetro total de los orificios }}{\text { Área total abierta }}=\frac{n * 2 \pi *\left(d_{o} / 2\right)}{n * 2 \pi *\left(d_{o} / 2\right)^{2}}=\frac{4}{d_{h}} \\
\beta=n *\left(\frac{d_{o}}{D}\right)^{2}
\end{gathered}
$$

Tabla 2. Geometría de flujo y parámetros de diseño.

\begin{tabular}{ccccccc}
\hline Prototipo & No. orificios & $\boldsymbol{d o}_{\boldsymbol{o}}(\mathbf{m m})$ & $\mathbf{D}(\mathbf{m m})$ & Área de flujo $\left(\mathbf{m m}^{\mathbf{2}}\right)$ & $\boldsymbol{\alpha}\left(\mathbf{m m}^{-1}\right)$ & $\boldsymbol{\beta}$ \\
\hline 1 & 3 & 4 & 25,4 & 37,70 & 1 & 0,074 \\
2 & 2 & 4 & 25,4 & 25,13 & 1 & 0,050 \\
\hline
\end{tabular}


Por otra parte, $\beta$ es un parámetro adimensional que relaciona el diámetro de los orificios con el diámetro del tubo y se plantea que tiene relación directa con el número de cavitación por autores como Ozonek [44]. $\beta$ generalmente es definido como el número de flujo, calculado a partir del número de orificios $n$, el diámetro del orificio $d_{o}$, y el diámetro de la tubería cilíndrica del reactor de cavitación, descrito en la ecuación 5 . Se observa que a medida que el número de flujo disminuye, se incrementa el grado de remoción en el sistema, resultado que puede ser atribuido al porcentaje de reducción del área de la placa perforada; para el prototipo número 2 , el porcentaje de remoción de área fue $90 \%$ y, de acuerdo a lo anteriormente señalado, este hecho hace que mejore la intensidad de la cavitación del sistema. Por lo tanto, es de resaltar que al diseñar la placa perforada se debe calcular el valor de $\beta$ para obtener mayor degradación de los contaminantes del fluido.

El rendimiento de la cavitación (cavitation yield) es de igual manera estudiado. Este parámetro considera la cantidad de sulfuro removido dividido entre la densidad de la potencia aplicada a cada sistema. En otras palabras, se define como el alcance de la degradación por energía consumida [45], y se representa $\sigma_{y}$ en $\mathrm{g} \mathrm{J}^{-1}$. La ecuación 6 se emplea para calcular el rendimiento de la cavitación. En esta ecuación la cantidad de sulfuro degradado se expresa en gramos, $H$ es el cabezal de presión del líquido en $\mathrm{m}, Q_{m}$ es el caudal medio en $\mathrm{m}^{3} \mathrm{~s}^{-1} \mathrm{y} t$ es el tiempo de reacción en s [37]. En la Tabla 3 se observan los resultados del cálculo del rendimiento de cavitación para los ensayos realizados en los dos prototipos. Acorde con Sivakumar y Pandit [46] a mayor número de orificios y menor área de cada uno de ellos se incrementa el rendimiento de la cavitación debido al perímetro genera en el líquido promueve la implosión súbita de las microburbujas en el sistema.

$$
\sigma_{y}=\frac{\text { Cantidad de sulfuro degradado }}{H \rho g Q_{m} t}
$$

Tabla 3. Rendimiento de cavitación para cada ensayo.

\begin{tabular}{cccc}
\hline Prototipo & $\begin{array}{c}\text { pH inicial del } \\
\text { agua residual }\end{array}$ & $\begin{array}{c}\text { Remoción de sulfuro } \\
\mathbf{( g )}\end{array}$ & $\begin{array}{c}\text { Rendimiento de } \\
\text { cavitación } \\
\left(\mathbf{g ~ J} \mathbf{~ J}^{-1} \mathbf{)}\right.\end{array}$ \\
\hline \multirow{2}{*}{1} & 10,0 & 11,48 & 2,955 \\
& 14,0 & 31,55 & 8,120 \\
2 & 10,0 & 297,60 & 0,028 \\
& 14,0 & 322,41 & 0,030 \\
\hline
\end{tabular}

\section{Beneficios secundarios de la técnica}

El agua residual procedente de la etapa de pelambre o decapado de la piel presenta altas cantidades de materia orgánica, pelo y sangre [47]; según Ozonek [31] y otras referencias bibliográficas $[48,49]$, el proceso de cavitación hidrodinámica da lugar a la formación de radicales $\mathrm{OH}^{*}$. Estos por su gran potencial oxidante pueden participar en la oxidación de sulfuros y de materia orgánica en el agua. De este modo el proceso beneficia la disminución de la demanda química de oxígeno en el agua. Sin embargo, al tener que emplear los radicales formados para remover otros contaminantes, demora más tiempo en remover la cantidad deseada de sulfuros. Adicionalmente, la cavitación da lugar a la homogeneización del tamaño de partículas en el agua residual, hecho que favorece la posterior clarificación por coagulación y flotación que se induce en el sistema (CAF) debido al tamaño de la burbuja formada en el cavitador [48]; la técnica CAF es de gran utilidad puesto que el lodo que se genera es mucho más compacto, facilitando su almacenamiento y disposición por la disminución de volumen que se presenta. Además, para la operación de la planta de tratamiento de aguas residuales, facilita la limpieza del tanque y remoción del lodo.

\section{Costo energético del tratamiento}

La cavitación hidrodinámica presenta ventajas tanto en la remoción de contaminantes, clarificación del agua residual industrial, como en la operación y funcionamiento del sistema de tratamiento. No obstante, es importante verificar que la técnica presente una ventaja económica frente al tratamiento actual para que sea sostenible en la industria. Por lo cual, en la Tabla 4, se presentan los costos estimados por cada uno de los 
experimentos realizados utilizando el tratamiento convencional y cavitación hidrodinámica en las variaciones presentadas, que; en total, son 5 resultados. Previamente, se ha descrito que el mejor resultado aplicando la técnica propuesta $(\mathrm{CH})$, se obtiene para el ensayo para el cual la potencia de la bomba fue $1471 \mathrm{~W}$ y un valor de $\mathrm{pH}$ del medio igual a 14,0 , dato que será de suma importancia para establecer que tan viable es en comparación al tratamiento convencional.

Para establecer el costo de tratamiento se deben evaluar bastantes factores, como el montaje del sistema, la capacidad de la bomba, los aditivos químicos, el personal operativo, entre otros. Sin embargo, para el presente estudio, el cálculo del costo unitario está representado por la energía consumida durante el tiempo de reacción. De esta manera el costo unitario es el producto de la energía eléctrica consumida durante el tratamiento multiplicado por el valor de la energía del lugar de operación, dividido por la masa de sulfuro removido en cada experimento (ecuación 7).

$$
\text { Costo unitario }\left(\frac{\$ U S D}{K g S^{2-}}\right)=\frac{(\text { tiempo de reacción }(h) * \text { potencia }(K w)) * \text { costo energía eléctrica } \frac{\$ U S D}{K w h}}{\text { sulfuro removido }\left(K g S^{2-}\right)}
$$

A pesar que el tratamiento por cavitación hidrodinámica no supera la efectividad del tratamiento convencional por oxidación catalítica en términos de oxidación de sulfuros en el agua, para un mismo periodo de reacción, es importante resaltar que el costo de remoción de sulfuros por cavitación es de 0,438 $\$ U S D / K g$ de $S^{2-}$ removido, es decir, $198 \%$ más bajo que el costo por el sistema convencional. Además, es preciso resaltar que el tratamiento convencional requiere la adición de un catalizador, el cual aumenta de forma considerable el costo unitario del sistema de tratamiento empleado.

Tabla 4. Costo unitario para la remoción de sulfuros por ensayo evaluado.

\begin{tabular}{|c|c|c|c|c|c|c|}
\hline Tecnología & $\begin{array}{c}\text { Potencia } \\
\text { (kW) }\end{array}$ & pH & $t(h)$ & $\begin{array}{l}\text { Consumo de } \\
\text { energía }(k W / h)\end{array}$ & $\begin{array}{c}\text { Sulfuro } \\
\text { removido (g) }\end{array}$ & $\begin{array}{l}\text { Costo unitario } \\
\text { (\$USD/kg) }\end{array}$ \\
\hline Tratamiento convencional & 5,22 & 14,0 & 1,5 & 7,83 & 578,0 & 0,86 \\
\hline \multirow{4}{*}{ Cavitación hidrodinámica } & 0,37 & 10,0 & 1,5 & 0,56 & 11,4 & 3,11 \\
\hline & 0,37 & 14,0 & 1,5 & 0,56 & 31,5 & 1,13 \\
\hline & 1,471 & 10,0 & 1,5 & 2,21 & 297,6 & 0,47 \\
\hline & 1,471 & 14,0 & 1,5 & 2,21 & 322,4 & 0,43 \\
\hline
\end{tabular}

* Referencia del costo de la energía de Colombia (2018) 0,064 \$USD/kWh.

\section{Conclusiones}

La disminución de la concentración de sulfuro de las aguas residuales producidas por el proceso de decapado o pelambre requerido en el proceso de curtido de pieles fue evaluada utilizando únicamente cavitación hidrodinámica, en comparación al tratamiento convencional que se realiza en la actualidad en dichas industrias.

La remoción de sulfuros del agua residual de pelambre empleando la tecnología de cavitación hidrodinámica es una alternativa efectiva para el tratamiento de las aguas residuales de curtiembre, en primer lugar porque la eficiencia del proceso de remoción es mayor a $\mathrm{pH}$ muy alcalino, como es el caso de las aguas residuales de pelambre hecho que facilita su tratamiento. Adicionalmente la diferencia de presión suministrada en cada prototipo empleado demuestra que la energía aplicada al sistema es completamente relevante en términos de remoción de sulfuro. Sin embargo, no es necesario contar con presiones sumamente altas como se puede observar en los resultados de los ensayos realizados con el prototipo 1 . Aunque la potencia de la bomba es baja, la configuración de las características de diseño y los factores hidráulicos permiten que alcance porcentajes de remoción bastante cercanos a los obtenidos por los ensayos del prototipo 2, el cual tenía una presión suministrada bastante mayor. Eso 
confirma que dichos parámetros del sistema y factores hidráulicos como el número de cavitación, el número de ciclos y los parámetros $\alpha$ y $\beta$ definidos por la cantidad y tamaño de los orificios ubicados en la placa son determinantes en el diseño del reactor. Por consiguiente, en la eficiencia del equipo, es notorio el marcado efecto que tiene la intensidad de la cavitación del equipo, variable que a medida que aumenta, hace mas eficiente la oxidación de sulfuro en el medio. Por otra parte, el efecto CAF generado por el equipo favorece la rápida clarificación del agua y la homogeneización de los sólidos presentes en el.

\section{Referencias bibliográficas}

[1] NPCS and NIIR-Project Consultancy Services, Leather processing and taining technology handbook. India: National Institute of Industrial Research; 2005.

[2] Di laconi C, Lopez A, Ramadori R, Di Pinto AC, Passino R. Combined chemical and biological degradation of tannery wastewater by a periodic submerged filter (SBBR). Water res. 2002;36(9):2205-14.

[3] Elsheikh MAS. Tannery wastewater pretreatment. Water Sci. Technol. 2009;60(2):43340.

[4] Schrank SG, José HJ, Moreira RFPM, Schröder HF. Applicability of Fenton and $\mathrm{H} 2 \mathrm{O} 2 / \mathrm{UV}$ reactions in the treatment of tannery wastewaters. Chemosphere. 2005;60(5):64455.

[5] Ganesh R, Balaji G, Ramanujam RA. Biodegradation of tannery wastewater using sequencing batch reactor-Respirometric assessment. Bioresour. Technol. 2006;97(15):1815-21.

[6] Organización Panamericana de la Salud and Ministerio de Salud. Lineamiento para la vigilancia sanitaria y ambiental del impacto de los olores ofensivos en la salud y calidad de vida de las comunidades expuestas en áreas urbanas. Conv. Coop. técnica No.485/10, 2012, 160.

[7] Secretaría Distrial de Ambiente. Guía de producción más limpia para el sector curtiembres de Bogotá. Enfoqueen vertimientos y residuos. Bogotá D.C., Colombia: Secretaría Distrial de Ambiente; 2017.
[8] Artuz LA, Martínez MS, Morales CJ. Las industrias curtiembres y su incidencia en la contaminación del río Bogotá. Isocuanta. 2011;1(1):43-53.

[9] Martínez S, Romero J. Revisión del estado actual de la industria de las curtiembres en sus procesos y productos: un análisis de su competitividad. Rev. Fac. Cienc. Econ. 2018;26(1):113-124.

[10]Fischer G, Miranda D, Carrarnza C, Rojas C, Jerez C, Zurita J. Accumulation of heavy metals in soil and plants of four vegetable crops irrigated with water of Bogotá river. Rev. Colomb. Cienc. Hortícolas. 2008;2(2):180-91.

[11] Salas G. Eliminación de sulfuros por oxidación en el tratamiento del agua residual de una curtiembre. Rev. Peru. Química Ing. Química. 2005;8(1):49-54.

[12] Olcay Tünay, Isik Kabdasli, Idil Arslan-Alaton, and Tugba Ölmez-Hanci, Chapter 3: Leather tanning industry, First Edit.). London, 2010.

[13] Ministerio de Ambiente, Vivienda y Desarrollo Territorial. Guia Ambiental para la Industria del Curtido y Preparado de Cueros. Bogotá D.C., Colombia: El Ministerio; 2006.

[14] Yu MH, Tsunoda H. Environmental toxicology: biological and health effects of pollutants. crc press; 2004

[15] Cadena F, Peters RW. Evaluation of chemical ozidizers for hydrogen sulfide control. Water Pollut. Control Fed. 1988;60(7):1259-63.

[16]Díaz-martínez JA, Granada-torres CA. Efecto de las actividades antrópicas sobre las características fisicoquímicas y microbiológicas del río Bogotá a lo largo del municipio de Villapinzón , Colombia. Rev. Fac. Med. 2018;66(1):45-52.

[17]Corporación Autónoma Regional de Cundinamarca - CAR, Acuerdo 16 de 1998, 16). COLOMBIA: OBSERVATORIO AMBIENTAL CAR, 1998, 38.

[18]Bagal MV, Gogate PR. Degradation of diclofenac sodium using combined processes based on hydrodynamic cavitation and heterogeneous photocatalysis. Ultrason. Sonochem. 2014;21(3):1035-43.

[19]Wang X, Zhang Y. Degradation of alachlor in aqueous solution by using hydrodynamic cavitation. J. Hazard. Mater. 2009;161(1):2027. 
[20] Patil PN, Gogate PR. Degradation of methyl parathion using hydrodynamic cavitation: Effect of operating parameters and intensification using additives. Sep. Purif. Technol. 2012;95:172-9.

[21] Parsons S. Advanced Oxidation Processes for Water and Wastewater Treatment. London: IWA; 2015.

[22] Wang X, Jia J, Wang Y. Combination of photocatalysis with hydrodynamic cavitation for degradation of tetracycline. Chem. Eng. J. 2017;315:274-82.

[23] Shah YT, Pandit AB, Moholkar VS. Cavitation Reaction Engineering. New York: Dan Luss series; 1999.

[24]Padoley KV, Saharan VK, Mudliar SN, Pandey RA, Pandit AB. Cavitationally induced biodegradability enhancement of a distillery wastewater. J. Hazard. Mater. 2012;219:6974.

[25]Flint EB, Suslick KS. The temperature of cavitation. Science. 1991;253(5026):1397-9.

[26] Saharan VK, Badve MP, PanditAB. Degradation of Reactive Red 120 dye using hydrodynamic cavitation. Chem. Eng. J. 2011;178:100-7.

[27] Chakinala AG, Bremner DH, Gogate PR, Namkung KC, Burgess AE. Multivariate analysis of phenol mineralisation by combined hydrodynamic cavitation and heterogeneous advanced Fenton processing. Appl. Catal. B Environ. 2008;78(1-2):11-8.

[28] Gogate PR, Bhosale GS. Comparison of effectiveness of acoustic and hydrodynamic cavitation in combined treatment schemes for degradation of dye wastewaters. Chem. Eng. Process. Process Intensif. 2013;71:59-69.

[29]Badve M, Gogate P, Pandit A, Csoka L. Hydrodynamic cavitation as a novel approach for wastewater treatment in wood finishing industry. Sep. Purif. Technol. 2013;106:15-21.

[30]Franke M, Braeutigam $P$, Wu ZL, Ren $Y$, Ondruschka B. Enhancement of chloroform degradation by the combination of hydrodynamic and acoustic cavitation. Ultrason. Sonochem. 2011;18(4):888-94.

[31] Ozonek J. Application of Hydrodynamic Cavitation in Environmental Engineering. CRC Press; 2012.

[32] Gogate PR, Kabadi AM. A review of applications of cavitation in biochemical engineering/biotechnology. Biochem. Eng. J. 2009;44(1):60-72.
[33] Balasundaram B, Harrison STL. Optimising orifice geometry for selective release of periplasmic products during cell disruption by hydrodynamic cavitation. Biochem. Eng. J. 2011;54(3):207-9.

[34]Amin LP, Gogate PR, Burgess AE, Bremner $\mathrm{DH}$. Optimization of a hydrodynamic cavitation reactor using salicylic acid dosimetry. Chem. Eng. J. 2010;156(1):165-9.

[35] Bis M, Montusiewicz A, Ozonek J, PasiecznaPatkowska S. Application of hydrodynamic cavitation to improve the biodegradability of mature landfill leachate. Ultrasonics sonochemistry. 2015;26:378-87.

[36] Clesceri LS, Rice EW, Baird RB, Eaton AD. Standard Methods for the Examination of Water and Wastewater. 22 ed. American Public Health Association, American Water Works Association, Water Environment Federation; 2012.

[37] Sivakumar M, Pandit AB. Wastewater treatment: A novel energy efficient hydrodynamic cavitational technique. Ultrason. Sonochem. 2002;9(3):123-31.

[38] Garcia B, Takarada T. Ca ion-exchanged coal char as H 2 S sorbent. Fuel. 1999;78(5):57381.

[39] Wang X, Wang J, Guo P, Guo W, Wang C. Degradation of rhodamine $B$ in aqueous solution by using swirling jet-induced cavitation combined with $\mathrm{H} 2 \mathrm{O} 2$. J. Hazard. Mater. 2009;169(1-3):486-91.

[40] Thanekar P, Panda M, Gogate PR. Degradation of carbamazepine using hydrodynamic cavitation combined with advanced oxidation processes. Ultrason. Sonochem. 2018;40(June 2017):567-76.

[41] Šarc A, Stepišnik-perdih T, Petkovšek M. The issue of cavitation number value in studies of water treatment by hydrodynamic cavitation. Ultrason. Sonochem. 2017;34:51-9.

[42] Parsa JB, Zonouzian SAE. Optimization of a heterogeneous catalytic hydrodynamic cavitation reactor performance in decolorization of Rhodamine B: Application of scrap iron sheets. Ultrason. Sonochem. 2013;20(6):1442-9.

[43] Huang Y, Wu Y, Huang W, Yang F, Ren XE. Degradation of chitosan by hydrodynamic cavitation. Polym. Degrad. Stab. 2013;98(1):37-43.

[44] Parag R, Gogate and Aniruddha B, Pandit. 
Engineering design methods for cavitation reactors II: Hydrodynamic cavitation. AIChE J. 2000;46(8):1641-9.

[45] Barik AJ, Gogate PR. Degradation of 4-chloro 2-aminophenol using a novel combined process based on hydrodynamic cavitation, UV photolysis and ozone. Ultrason. Sonochem. 2016;30:70-8.

[46]Kirpalani DM, McQuinn KJ. Experimental quantification of cavitation yield revisited: Focus on high frequency ultrasound reactors. Ultrason. Sonochem. 2006;13(1):1-5.
[47] Salas G. Eliminación de sulfuros por oxidación en el tratamiento del agua residual de una curtiembre. Rev. Peru. Química Ing. Química. 2005;8(1):49-54.

[48] Gągol M, Przyjazny A, Boczkaj G. Wastewater treatment by means of advanced oxidation processes based on cavitation - A review. Chem. Eng. J. 2018;338(September 2017):599-627.

[49] Jyoti KK, Pandit AB. Effect of cavitation on chemical disinfection efficiency. Water Res. 2004;38(9):2248-57. 